Document downloaded from:

http://hdl.handle.net/10251/149083

This paper must be cited as:

Yamanaka, E.; Tortajada-Genaro, LA.; Maquieira, A. (2017). Low-cost genotyping method based on allele-specific recombinase polymerase amplification and colorimetric microarray detection. Microchimica Acta. 184(5):1453-1462. https://doi.org/10.1007/s00604-017-2144-0

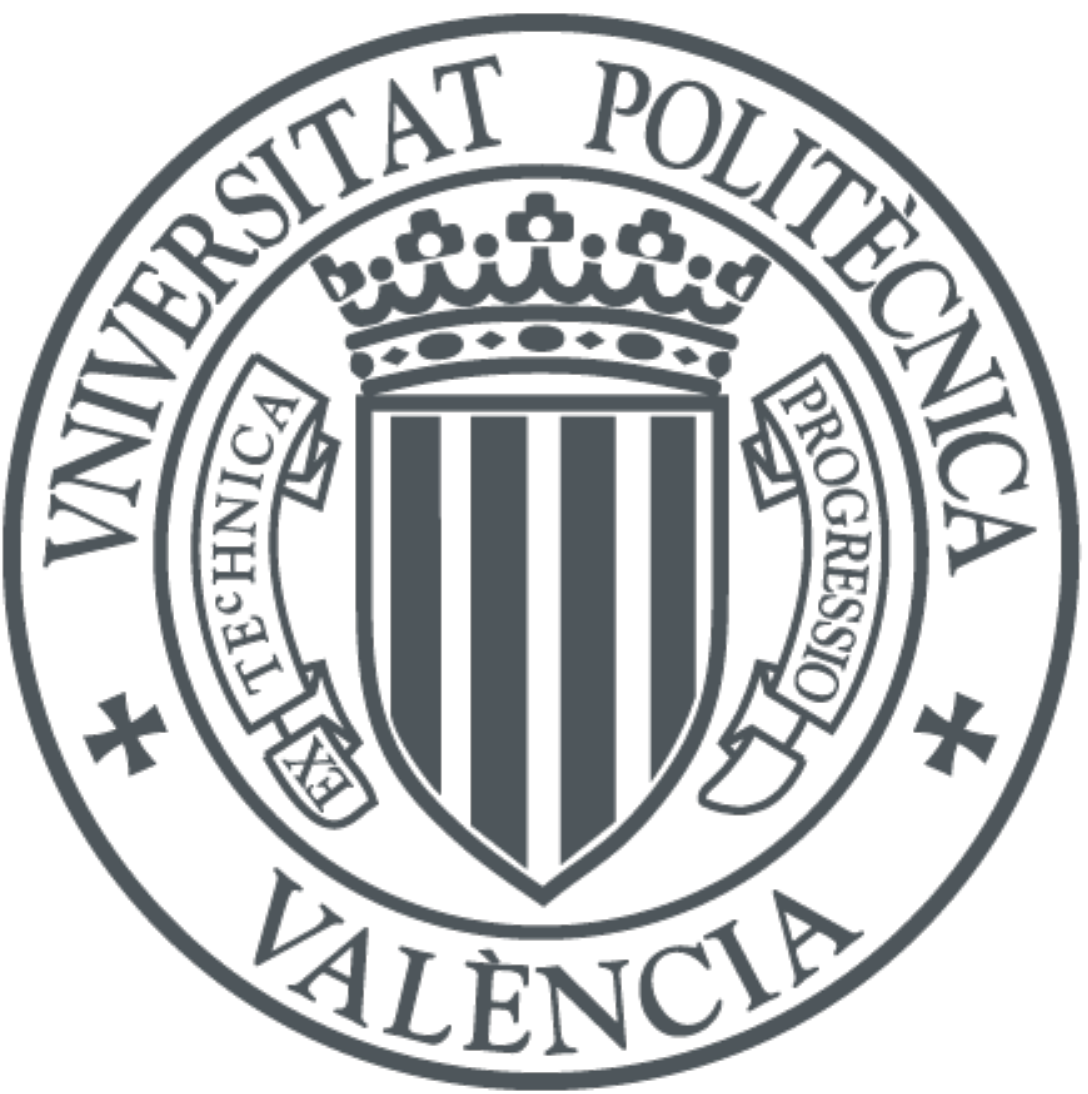

The final publication is available at

https://doi.org/10.1007/s00604-017-2144-0

Copyright Springer-Verlag

Additional Information 


\section{Low-cost genotyping method based on allele-specific recombinase polymerase amplification and colorimetric microarray detection}

Eric Seiti Yamanaka ${ }^{(a)}$, Luis A. Tortajada-Genaro(a,b), Ángel Maquieira ${ }^{(a, b)}$

(a) Instituto Inter-universitario de Reconocimiento Molecular y Desarrollo Tecnológico (IDM) - Departamento de Química, Universitat Politècnica de València, Camino de Vera s/n, E46022 Valencia, Spain

(b) Unidad Mixta UPV-La Fe, Nanomedicine and Sensors, Valencia, Spain

1 ABSTRACT

2 The costs of current genotyping methods limit their application to personalized

3 therapy. The authors describe an alternative approach for the detection of

4 single-point-polymorphisms (SNPS) using recombinase polymerase

5 amplification (RPA) as an allele-specific technique. The use of short and

6 chemically modified primers, locked nucleic acids (LNA), allowed the selective

7 isothermal amplification of wild-type or mutant variants at $37^{\circ} \mathrm{C}$ in $40 \mathrm{~min}$. An

8 amplification chip containing 100 wells was manufactured with a 3D printer and

9 using thermoplastic polylactic acid (PLA). The platform reduces costs, reagent 10 consumption, sample volume and allows assay parallelization compared to 11 other amplification formats. As proof of concept, the method was applied to the 12 genotyping of four SNPs that are related to the treatment of tobacco addiction. 13 The target polymorphisms were rs4680 (COMT gene), rs1799971 (OPRM1 14 gene), rs1800497 (ANKK1 gene), and rs16969968 (CHRNA5 gene). The 15 capabilities of the method are demonstrated detecting the reaction products 16 using a colorimetric technique in microarray format. The genotype populations 17 can be well discriminated. 


$$
0^{20^{2}}
$$




\section{Graphical abstract}

22 A method based in the allele-specific recombinase polymerase amplification 23 was developed for the genotyping of polymorphisms. The isothermal 24 discrimination reaction was produced in a multi-well amplification chip 25 manufactured with a 3D printer and using thermoplastic polylactic acid.

26
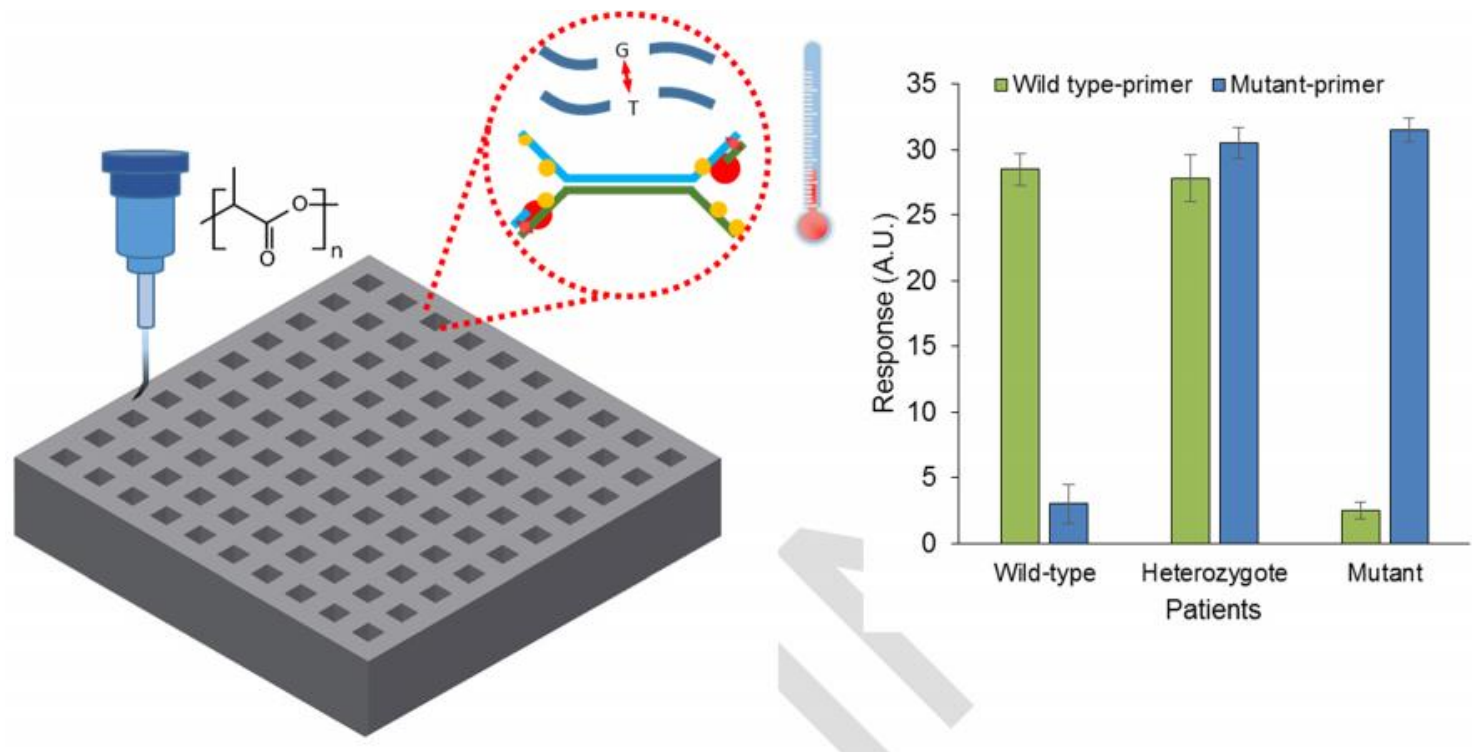

27

28 


\section{INTRODUCTION}

30 Pharmacogenomics is currently considered one of the most active areas of the 31 personalized medicine paradigm. However, numerous barriers have been encountered to launch DNA variation analyses, such as single-nucleotide polymorphisms (SNPs), in health systems [1]. Among other factors, available platforms have a marked technological limitation. Most genomic findings have been obtained from high-throughput technologies, such as Illumina and Affimetrix platforms. However, the incorporation of these methods into primary care centers is limited, and cost is the main drawback [2-4]. Dramatically cutting the turnaround times of these platforms is an important goal for implementing SNP testing into clinical scenarios. Therefore, the novel generation of simple diagnostic tools is absolutely necessary for the real adoption of personalized medicine $[5,6]$.

A large family of high-potential methods to be developed in simple systems is that based on allele-specific (AS) amplification [7]. Polymerase chain reaction (PCR) uses primer pairs, deliberately designed at SNP sites. Primers have a single-base variation at the 3 ' end (allele-specific primers), so extension and amplification reactions take place with only perfectly-matched sequences of target regions. This approach has been successfully used as a pharmacogenomic tool combined with several detection systems [8-10]. Nevertheless, these techniques require particular thermal cycling, consequently there are several limitations for their future integration as point-of-care devices. PCR demands an accurate temperature control system to quickly heat/cool reaction solutions. The high temperature reached (up to $95{ }^{\circ} \mathrm{C}$ ) leads to variations in the volume reaction due to water evaporation and gas bubble formation, which renders accurate process control necessary.

Several new technologies have emerged to improve DNA-based analyses [11]. Many efforts have been made that focus on developing low-cost systems to be used for point-of-care applications or in small laboratories located at the physician's office or in primary health centers.

59 A revolution in the development of new methods is currently being witnessed, 60 and is associated with the application of isothermal solutions for microanalyses 
61 [12]. These approaches are based on using proteins that separate DNA strands 62 instead of thermal approaches so that target nucleic acids are synthesized at 63 constant temperature. Nevertheless, the application of isothermal amplification 64 for SNP genotyping is still minimum [13,14]. Among isothermal reactions, recombinase polymerase amplification (RPA) has many interesting properties; e.g., short reaction times (20-60 $\mathrm{min}$ ), robustness and low energy requirements (close to room temperature) [15]. Recently, our research group has demonstrated that polycarbonate-based substrates can be used to support RPA assays in chip [16], micro-reactors [17], or dynamic formats [18]. A well array chip has been described to process miniaturized RPA assays, applied for pathogen detection in under $30 \mathrm{~min}$ [19]. The chip was manufactured from a silicon substrate by a complex fabrication technique, including photolithography and chemical treatment, and is only available in specialized laboratories.

74 The capability of RPA technology for SNP genotyping remains an unsolved issue. A recent study evaluated the influence of sequence mismatches on the amplification specificity of closely-related pathogens [20]. A proof of concept assay describes the discrimination of a single-point mutation of the HRAS gene [21]. To this end, DNA extracted from cell lines was selectively amplified and genetic variants were distinguished by measuring the wavelength shift on silicon micro-ring resonators. However, this technology is far from being adopted generally in health centers.

82 In the present study, the capability of allele-specific RPA (AS-RPA) is evaluated 83 and a low-cost method is developed for the SNP genotyping of clinically 84 relevant polymorphisms. In a first approach, amplification is performed in conventional polypropylene vials in a single format (detection of a single polymorphism). The advantages of this disposable format are its low cost and compatibility with a huge number of laboratory equipment. However, lab-on-achip or $\mu$-total analysis systems offer important advantages for diagnostic devices, such as high-throughput and miniaturization, among others [22].

90 There are many ways to microfabricate plastic-based materials; e.g. laminate, 91 embossing or injection molding. Additive manufacturing is being examined given its growing interest in the microfluidics field [23]. It is capable of producing 
customized structures that range from a few microns to several centimeters in a

94 single step. The main limitations of 3D printers are related to spatial resolution, dimensional fidelity, surface quality, biocompatibility, optical transparency, among others [24]. Advantages include low infrastructure costs and easy manufacturing compared to photolithography or soft lithography approaches. In order to evaluate the potential of this technology, a well array chip for performing AS-RPA was designed and developed with a commercial 3D printer.

100 The objective was to demonstrate RPA's capability as a genotyping method and 101 to compare its performance in an advanced platform compared to the standard 102 format (vials).

103 As proof of concept, the solution was applied as a pharmacogenomics tool to 104 treat smoking cessation and the highly addictive properties of nicotine [25,26]. 105 In such diseases, the benefits of personalized medicine based on genotype 106 populations has been demonstrated. However, the high cost of current 107 genotyping technologies, compared to the cost of ineffective or erroneous 108 treatment, compromises the application of the test. Therefore, the approach was designed by following the analytical quality and health system sustainability goals.

\section{MATERIAL AND METHODS}

\section{Target genes}

114 The target polymorphisms for the tobacco use disorder were rs4680 (COMT gene), rs1799971 (OPRM1 gene), rs1800497 (ANKK1 gene) and rs16969968

116 (CHRNA5 gene). The wild-type variants are $\mathrm{G}, \mathrm{A}, \mathrm{G}$, and $\mathrm{G}$, and the mutant 117 variants are $A, G, A$, and $A$, respectively. The pharmacogenomic information 118 about these variants is included as Supplementary Material (Tables SI.1, SI.2 119 and SI.3). The human beta actin (ACTB) gene was selected as an endogenous 120 control. The list of oligonucleotides for the genotyping of each SNP is found in 121 Table SI.4. 


\section{Patient samples and reference discrimination method}

125 Subjects $(n=17)$ were recruited for the present study according to ethics and 126 with informed consents. Buccal smear samples were collected using sterile 127 swabs. They were submitted to digestion and purification steps with a PureLink 128 Genomic DNA Mini Kit (Invitrogen, Thermo Fisher Scientific, USA, 129 www.thermofisher.com). The isolated DNA extracts were eluted from the spin130 columns of the kit with Tris- $\mathrm{HCl}$ buffer ( $10 \mathrm{mMTris}, \mathrm{pH}$ 8.6) and stored at $-20^{\circ} \mathrm{C}$ 131 until analyzed.

132 Allele-specific PCR in a single format was applied as the reference genotyping 133 method. Briefly, the extracted genomic DNA (4 ng) was amplified using two 134 PCR master mixes (Biotools, Spain, www.biotools.eu) and employing $300 \mathrm{nM}$ of each variant primer pair (reverse and allele-specific forward). To confirm amplification, products were diluted in 0.5x SyBR Safe (Invitrogen, Thermo

137 Fisher Scientific, USA) and submitted to fluorescence measurements in a plate 138 reader (Victor 3TM V1420, PerkinElmer, Finland, www.perkinelmer.com). 139 Genotypes were also verified by 3\% agarose gel electrophoresis, followed by 140 dying with an intercalating agent (Real Safe, Durviz, Spain, www.durviz.com) and visualization under UV light.

\section{Fabrication of structured platforms}

144 The 100-well array chip was modeled with the Autodesk Inventor Professional 1452015 software (Autodesk, USA, www.autodesk.com) and fabricated with a 3D 146 printer (Ultimaker 2 Extended, UltimakerB.V., the Netherlands, 147 www.ultimaker.com). Polylactic acid (PLA) filament (RS Pro, Spain; $2.85 \mathrm{~mm}$ 148 diameter, www.rs-online.com) was employed as the printing material. 149 Fabrication was carried out using a $0.4 \mathrm{~mm}$ diameter nozzle at $210^{\circ} \mathrm{C}$ and a 150 bed operation temperature of $60^{\circ} \mathrm{C}$. In order to evaluate the best printing conditions, prototypes were fabricated with different layer thicknesses (up to 0.2 $152 \mathrm{~mm}$ ) and at various printing speeds (up to $300 \mathrm{~mm} \cdot \mathrm{s}^{-1}$ ). Subsequently, printed 
153 structures were cleaned with a 30-minute ultrasonic bath and dried with 154 compressed air.

155 The fabrication quality of the PLA-chips was monitored by optical microscopy 156 imaging. Surface pictures were captured (1.2x magnification) by an Olympus 157 SZ61 stereo microscope (Olympus Co., Japan, www.olympus.com). Images were analyzed with the Image $\mathrm{J}$ software to provide an estimated roughness for each sample. Surface hydrophobicity was estimated from the contact angle data. Measures of the deionized water droplets $(4 \mu \mathrm{L})$ were taken using a DinoLite Digital Microscope (AnMo Electronics Co., Taiwan, www.dino-lite.com) at the 1.3 megapixel resolution.

A mass loss study was also done to evaluate possible sample evaporation on the platform. The chip was loaded with 10 RPA samples ( $4 \mu \mathrm{L}$ each) and placed in an oven at $37^{\circ} \mathrm{C}$. Mass measurements of the set were periodically taken during $4 \mathrm{~h}$ and compared with those of an unloaded reference chip.

\section{Assay protocol: amplification}

169 The amplification step was performed using a TwistAmp Basic RPA kit 170 (TwistDx, UK, www.twistdx.co.uk). Eight allele-specific mixes (2 per SNP) were 171 prepared with rehydration buffer, $14 \mathrm{mM}$ of magnesium acetate, $480 \mathrm{nM}$ of 172 allele-specific forward primer and reverse digoxigenin-labeled primer, and the 173 enzyme pellet. Mineral oil (8\%) was also added to minimize sample 174 evaporation. Solutions were loaded onto the 100-well array chip and the DNA 175 template (2.56 $\mathrm{ng}$ ) was added to allow the simultaneous amplification of eight 176 different allelic variants for 10 patient samples and controls (human ACTB 177 gene). The chip was then covered with a polyester plate sealer (Corning, USA, 178 www.corning.com) and gently vortexed to mix reagents and samples. 179 Amplification was carried out in a heating oven (Memmert UF30, Germany, 180 www.memmert.com) at $37^{\circ} \mathrm{C}$ for $40 \mathrm{~min}$.

181 The AS-RPA reactions were also performed in $0.2 \mathrm{~mL}$-polypropylene vials 182 (Labbox, Spain, www.labbox.com) and polycarbonate home-made array chips. 183 These chips were fabricated using a computer numerical control drilling 
184 machine (Bungard CCD, Karo 5410, Germany, www.bungard.de). The feed 185 speed and rotational rate of the drill were respectively $2000 \mathrm{~mm} \mathrm{~s}^{-1}$ and 48000 $186 \mathrm{rpm}$. The diameter of each well was $5 \mathrm{~mm}$ and their depth was $1.1 \mathrm{~mm}$. The composition of the RPA mixtures was the same as that previously described, but volumes were $25 \mu \mathrm{L}$ and $4 \mu \mathrm{L}$ per reaction for vials and chips, respectively. The heating system used was a thermocycler (TC-4000, Techne, UK, www.techne.com) and an oven, respectively.

\section{Assay protocol: detection and data analysis}

193 The AS-RPA products were detected by a hybridization assay on polycarbonate 194 chips, adapted from reference [10]. Briefly, the mixtures of the wild-type or mutant products for all four SNPs were prepared from the respective single RPA solutions. For this purpose, $2 \mu \mathrm{L}$ of each amplification product were diluted in $16 \mu \mathrm{L}$ of hybridization buffer composed of $\mathrm{NaCl} 225 \mathrm{mM}$, sodium citrate 22.5 $\mathrm{mM}, 10 \%$ formamide and $2.5 x$ Denhardt's solution, $\mathrm{pH} 7$. Subsequently, mixtures were heated at $95{ }^{\circ} \mathrm{C}$ for $10 \mathrm{~min}$ for denaturation and transferred to the chips with the immobilized probes in the microarray format. After $60 \mathrm{~min}$ of incubation at $37^{\circ} \mathrm{C}$, chips were washed with diluted hybridization buffer. The immunoreaction protocol with enzymatic labelling was followed to develop the duplex of the probe-RPA product, as described in reference [10]. The oxidized form of 3,3,5,5-tetramethylbenzidine (substrate of horse-radish-peroxidase) produced a blue precipitate over the positive or control spots. Chips were then read with a desktop scanner (Epson Perfection 1640SU Office, Epson, Japan, www.epson.com).

208

\section{Discrimination index}

210 The resulting gray-scale images (Tagged Image File Format, color depth 16 bit) 211 were processed by an in-house software for the microarray analysis. The optical 212 intensity signals of each spot and local background were quantified by 213 generating a data matrix of the signal-to-noise ratios. The genotype 214 determination rule was constructed according to the replicated responses of the 
215 specific probes for each polymorphism. A discrimination index was calculated 216 from the signal of the wild-type (WT) and mutant (MUT) variants according to 217 this equation: (WT - MUT)/(WT + MUT). The Statgraphics Centurion statistical 218 package for Windows v.16 was used for the data analysis.

219

\section{RESULTS}

\section{RPA capability as a genotyping tool}

222 The use of RPA as an allele-specific amplification technique was analyzed by 223 considering the role of each element in the process. A recombinase (T4 uvsX) 224 recognizes targeted DNA templates and specific primers at a high affinity and 225 catalyzes subsequent homologous pairing and strand exchange [20]. 226 Polymerase produces the correct elongation of the perfect-annealed 227 primer/template, and is the key reaction in the DNA duplication process [27]. 228 Furthermore, the Pol I large fragment (Bsu polymerase) lacks exonuclease 229 activity $\left(3^{\prime} \rightarrow 5^{\prime}\right)$ that may modify the target nucleotide. Therefore, we expected 230 the presence of mismatches on their 3'-extreme to hamper the nonspecific 231 reaction due to the combined action of two enzymes, even at a low working 232 temperature.

233 Complementarily, oligonucleotide sets were carefully selected to satisfactorily 234 amplify/detect the given template region. The in silico design restrictions were 235 primer length, absence of secondary structures, and primer/template duplex 236 stability. Both these last parameters were estimated from the thermodynamic 237 models available for DNA duplexes [28]. Although the recommended length for 238 RPA primers should be $30-35$ bases long according to the manufacturer's 239 instructions, shorter primers (19-21 mer) were chosen to improve selectivity.

240 The free energy $(\Delta G)$ values for the self-annealing and hairpin structures were 241 restricted to $1.0 \mathrm{Kcal} \cdot \mathrm{mol}^{-1}$ (the equivalent to melting temperatures $<50{ }^{\circ} \mathrm{C}$ ). 242 The selected oligonucleotides produced primer/template duplexes with changes 243 in free energy $(\Delta G)$ of $-25.6 \pm 0.2 \mathrm{Kcal} \cdot \mathrm{mol}^{-1}$ (the equivalent to a melting 244 temperature of $75.4 \pm 0.1^{\circ} \mathrm{C}$ ) for totally complementary primers. The duplexes 
245 between the template and mismatched primers were less stable $(-24.0 \pm 0.6$ $\left.246 \mathrm{Kcal} \cdot \mathrm{mol}^{-1}, 72.7 \pm 0.6^{\circ} \mathrm{C}\right)$.

247 The experiments focused on evaluating discrimination capability using the 248 designed primers that differentiated at their 3'-endnucleotide. Figure 1a shows 249 the kinetic curve to perform amplification in a homogeneous format (reaction 250 volume of $25 \mu \mathrm{L}$ ). The expected positive signals were observed after 10-20 min 251 following typical logistic regression (maximum response after $60 \mathrm{~min}$ ). Under 252 the selected conditions, a different behavior was observed depending on the 253 added primer. Extension by polymerase was efficient when the 3' terminal base 254 of a primer matched its target, whereas extension was inefficient or nonexistent 255 when the terminal base was mismatched. These effects agree with the 256 previously reported results about the reduction or inhibition of the RPA reaction 257 due to the presence of a mismatch in the primer/template duplex [20].

(a)

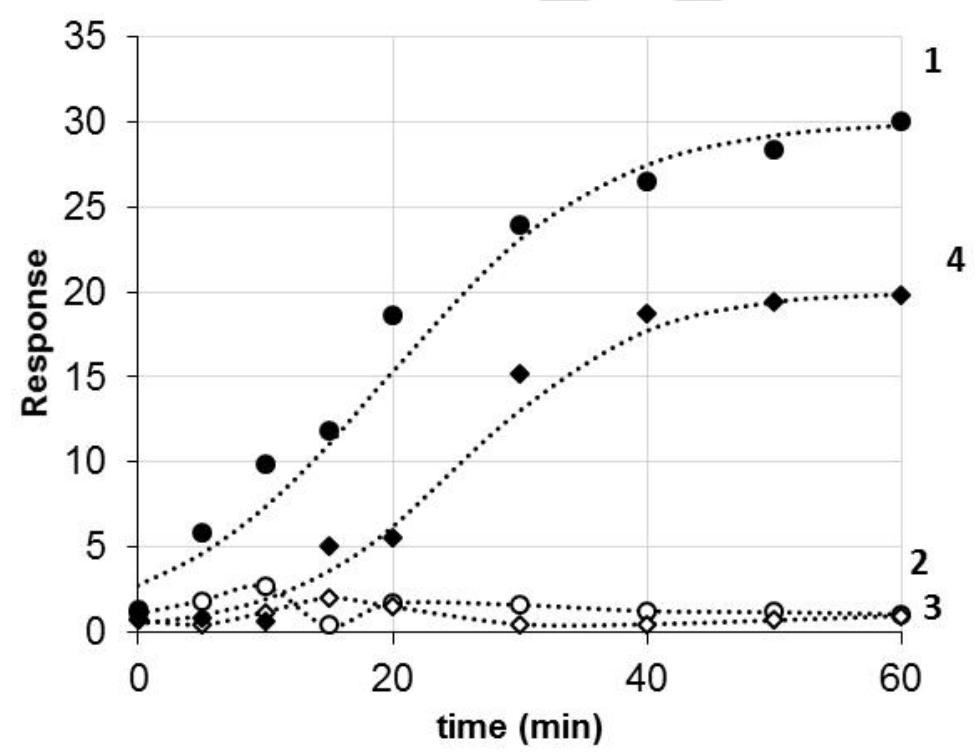




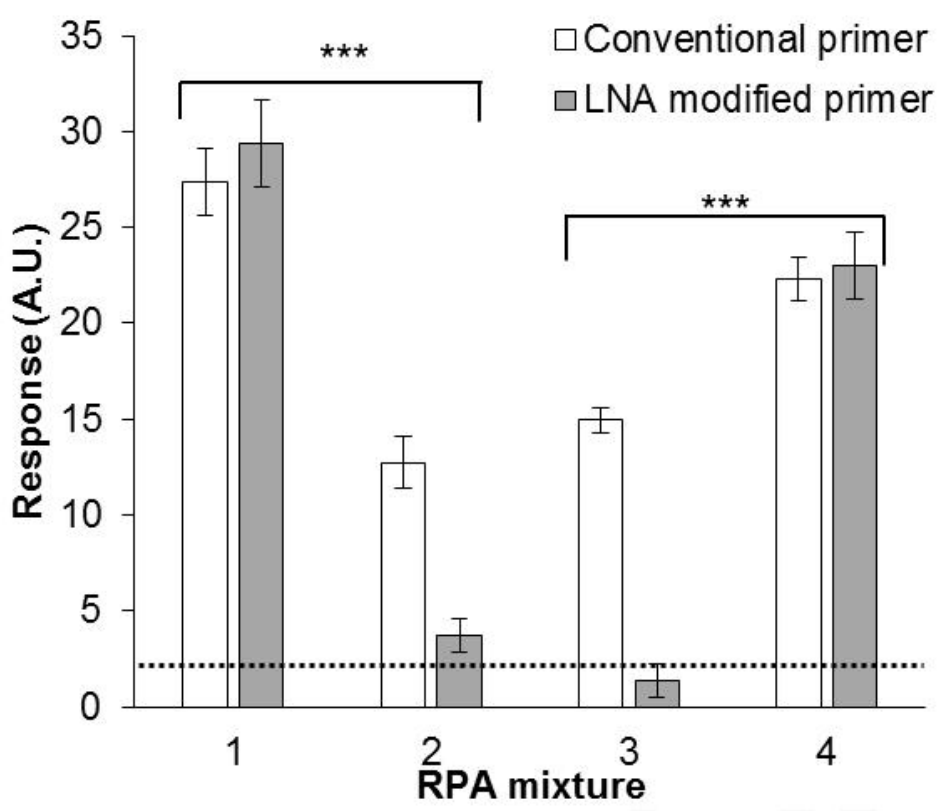

263 Figure 1. (a) Amplification kinetic curves of rs1799971 (OPRM1 gene) 264 depending on the RPA mixture: logistic regressions $y=30 /(1+\exp (-2.28-0.12$ $265 \mathrm{t}), \mathrm{R}=0.977$ for the wild-type and $\mathrm{y}=20 /(1+\exp (-3.64-0.15 \mathrm{t})), \mathrm{R}=0.977$ for 266 the mutant variant. (b) Response depending on the primer nature and RPA 267 mixture: statistical comparison compared to the perfect-match duplex (*** $268 \mathrm{p}<0.001)$. Mixture 1: wild-type template DNA and wild-type FP). Mixture 2: wild269 type template DNA and mutant FP. Mixture 3: mutant template DNA and wild270 type FP. Mixture 4: mutant template DNA and mutant FP. Four replicates

272 Conventional and chemical-modified primers, locked nucleic acids (LNA), were 273 compared for AS-RPA. Figure $1 \mathrm{~b}$ shows that nonspecific amplification took 274 place for the mismatched duplexes between the primer and templates. 275 Nevertheless, the amplification yield was significantly higher for the totally 276 complementary duplexes (ANOVA, p-value $<0.001$ ). With the LNA primers, 277 differences were more marked, and even nonspecific amplification was similar 278 to the negative controls. These experiments demonstrated that the presence of 279 this nucleoside at the 3' terminal base improved allelic discrimination.

280 A multiplex reaction was studied for the simultaneous amplification of more than 281 one target in a single reaction. However, reaction yields were not satisfactory 282 and there were sensitivity losses. One system displayed dominating and/or 
283 inhibiting activity over other primers and amplicons, and even genotyping 284 capability was lost. These results agreed with conventional RPA behavior and 285 can be associated with their high sensitivity to the total primer concentration 286 [16].

\section{Design and fabrication of well array chip}

289 The amplification assays, described in the previous section, were performed in 290 0.2-mL polypropylene vials. The next step was to reduce the reaction volume by 291 performing the assay in a well array chip. This kind of platforms improves 292 amplification capabilities, particularly high-throughput [29]. Fused filament 293 fabrication was chosen as the additive manufacturing technology, with a 294 biocompatible polymer, e.g., PLA, as the thermoplastic material. This technique 295 was selected because it produces innovative bioanalytical platforms that can be customer-designed and fast prototyped by a 3D printer.

297 The first experiments focused on designing an array chip with 100 wells. The 298 well dimensions $(2.5 \mathrm{~mm} \times 2.5 \mathrm{~mm} \times 4 \mathrm{~mm})$ were chosen to perform RPA in a 299 reduced volume $(<5 \mu \mathrm{L})$. Edge-to-edge spacing (distance between wells) was 1 $300 \mathrm{~cm}$ for RPA-mixture dispensation by a multi-channel micropipette. The 301 deposition of filament layers, one on top of the other, built up the bottom and the 302 walls of the chip. This additive technique produced grooved structured surfaces 303 on chip walls to study the effect of the 3D printing parameters on chip quality 304 (Supplementary Material/Figure SI.1). By increasing printing layer separation, 305 groove thickness changed from $71 \pm 2 \mu \mathrm{m}(0.06 \mathrm{~mm})$ to $238 \pm 5 \mu \mathrm{m}(0.2 \mathrm{~mm})$. 306 With a 0.02-mm layer height, the surface became irregular and did not produce 307 visible grooves. Surface roughness, expressed in Rq, varied from $53.8 \mu \mathrm{m}(0.02$ $308 \mu \mathrm{mm})$ to $76.6 \mu \mathrm{m}(0.2 \mathrm{~mm})$. The effect of printing speed and working 309 temperatures during the deposition process were negligible. The selected values were a layer height of $0.1 \mathrm{~mm}$ and a print speed of $50 \mathrm{~mm} \cdot \mathrm{s}^{-1}$, which

311 resulted in a fabrication time of 480 min.unit ${ }^{-1}$. Figures $2 a$ and $2 b$ show the 312 optical microscope images of the PLA-chips produced under the selected 313 conditions. Sealing, performed with a polyester adhesive film and by adding 
314 mineral oil, was effective for the tested range (up to $37^{\circ} \mathrm{C}$ and $4 \mathrm{~h}$ ), with null 315 leaking and evaporation ( $p$-value $<0.001$ ).

(a)

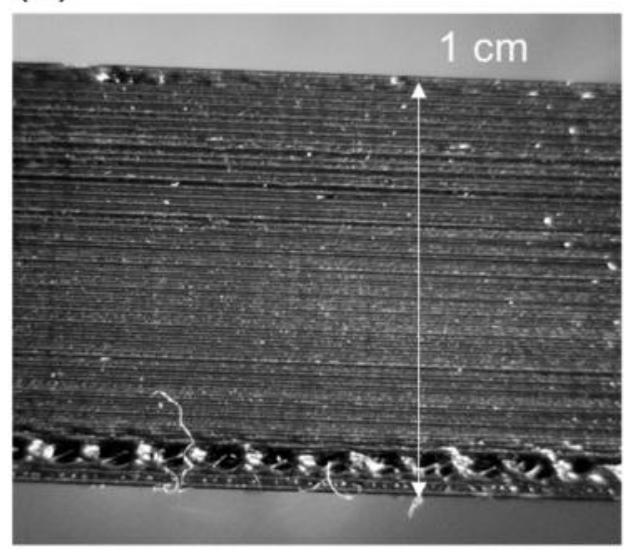

(b)

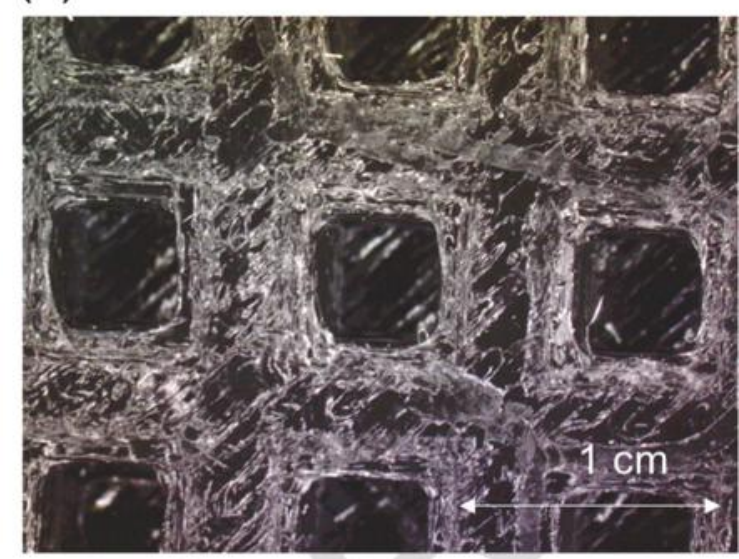

(c)

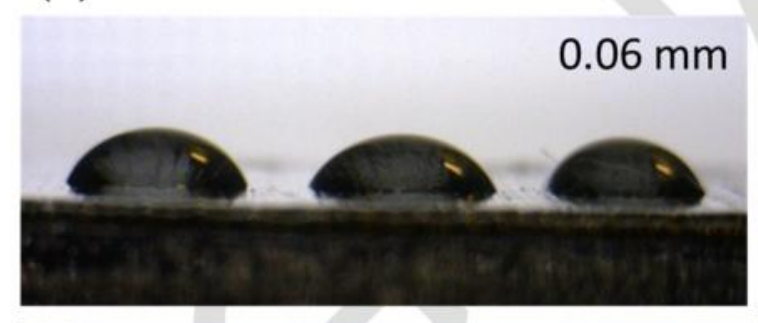

$0.2 \mathrm{~mm}$

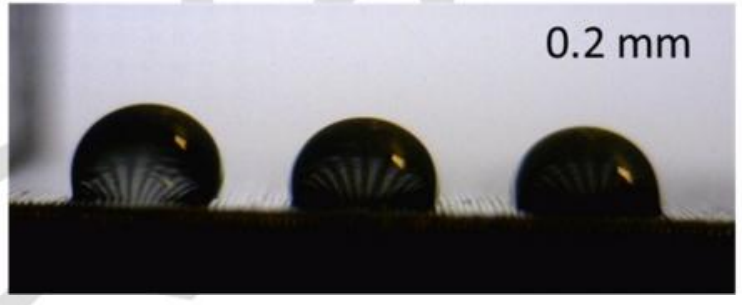

Figure 2. (a) Optical microscope image of the chip wall. (b) Optical microscope 318 image of chip wells (top view). (c) Effect of printing layer height on the PLA 319 contact angle

321 The hydrophilic/hydrophobic nature of the reaction vessel can affect the easy322 filling of wells. Hence the wettability of the raw PLA-chips was estimated and 323 the contact angle of well surfaces was measured. The results were $97 \pm 4^{\circ}$ and $32477 \pm 2{ }^{\circ}$ for the wall and the bottom surface, respectively. These values indicated 325 how the patterned topography modified the interfacial tension between the liquid 326 and thermoplastic compared to the base material (PLA, contact angle of about $32780{ }^{\circ}$ ). Two chemically modified chips (UV/ozone irradiation and PEG 
passivation) were tested. The surface topographies, estimated by microscope

329 image analyses, were comparable to those from the raw chip ( $p$-value $>0.05$ ).

330 After applying soft UV/ozone irradiation, the contact angle was $71 \pm 2^{\circ}$. This 331 lower value indicated that some photo-oxidized polar groups on the surface 332 were formed, consequently fiber adhesion increased (higher surface energy). 333 Addition of PEG also produced a coating over all the active surfaces, and 334 hydrophilicity increased $\left(62 \pm 3^{\circ}\right)$. Regardless of the surface treatment, reagent solutions were easily loaded in wells.

336 The effect of the unpolished surfaces and chemical treatment on the 337 amplification yield was studied. Replicate RPA reactions were performed using 338 genomic DNA for native/mutant patients (order of magnitude: $10^{3} \mathrm{pg}$ of gDNA). 339 Effective amplification was achieved in all the wells of the raw and chemically 340 modified PLA-chips. Nevertheless, the PEG coating was chosen because this 341 treatment can help block chip surfaces, and prevent nonspecific signals and sample losses through protein and amplification product adsorption [30].

\section{Comparison of amplification platforms}

345 The RPA performances for the reactions run in the PLA-3D printed chip were 346 compared with two previously reported platforms (Table 1 ). The first reference 347 platform was polypropylene vials $(0.2 \mathrm{~mL})$ (individual or tube strips), which are 348 widely used for DNA amplification in conventional thermal cyclers. The second 349 was micro-reactors fabricated in polycarbonate (PC) substrate by drilling 350 because they are a low-cost alternative for reduced volume amplifications 351 performed in ovens or other cheap thermal systems [17]. The analysis of variance (ANOVA) indicated that the responses for three platforms were comparable, with $p$-values of 0.63 for the negative controls, 0.27 for the 354 reference gene (ACTB gene) and 0.23 for the target genes. The platform cost 355 for 100-plex reactions using the current 3D-printed chips was the equivalent to 356 those of the polypropylene vials. Nevertheless, the main advantages of this approach stemmed from volume reduction and the cost of the reagent; reagent consumption (and the amount of DNA) decreased by about 6 -fold. Other advantages were reduced size, which was compatible with portable heating 
360 systems (i.e. miniaturized Peltier-based devices), and facilitated their adaptation 361 for field or doctor office applications [11,12]. These performances confirmed 362 PLA-additive manufacturing to be a strategy for the rapid versatile low-cost 363 prototyping of bioanalytical devices. The assay costs of each platform were 364 estimated, considering their material, equipment and processing expenses (3D 365 printing or CNC milling), as well as their number of parallel assays. The 366 estimated platform cost per assay for the 3D printed PLA chip was similar to 367 polystyrene vials, while 4-times lower than for the polycarbonate milled chips.

368 Our approach based on reaction vessels was compared with microfluidic chips 369 in virtue of their high applicability as point-of-care systems [31]. The microfluidic 370 platforms, generally based on poly(methyl-methacrylate) (PMMA) or similar 371 polymers, allow a higher degree of assay integration and lower reaction 372 volumes (nanoliter scale). In exchange, the PLA multi-well chip presents easier 373 manipulation, no fluidic control equipment requirements and a simpler 374 fabrication process with a 16-fold lower cost.

375 This study can open up ways to test PLA-microfluidic devices, e.g., integration 376 of RPA amplification and real-time detection, prior to their mass production in 377 other thermoplastics, such as PC (e.g., injection molding).

378

379

380 


\begin{tabular}{|c|c|c|c|}
\hline & PLA chip & PP vial & PC chip \\
\hline $\begin{array}{l}\text { Response negative } \\
\text { control }\end{array}$ & $3 \pm 2$ a.u. & $4 \pm 1$ a.u. & $4 \pm 1$ a.u. \\
\hline $\begin{array}{l}\text { Response reference } \\
\text { gene }\end{array}$ & $37 \pm 3$ a.u. & $41 \pm 2$ a.u. & $39 \pm 3$ a.u. \\
\hline $\begin{array}{l}\text { Response target } \\
\text { genes }\end{array}$ & $34 \pm 3$ a.u. & $37 \pm 1$ a.u. & $35 \pm 1$ a.u. \\
\hline $\begin{array}{l}\text { Fabrication } \\
\text { technique }\end{array}$ & 3D printing & Molding & Molding + Milling \\
\hline $\begin{array}{l}\text { Platform } \\
\text { dimensions }\end{array}$ & $\begin{array}{c}52 \mathrm{~mm} \times 52 \mathrm{~mm} \times 10 \\
\mathrm{~mm}\end{array}$ & $100 \times(20 \mathrm{~mm}, \phi 7 \mathrm{~mm})$ & $\begin{array}{c}30 \mathrm{~mm} \times 30 \mathrm{~mm} \times 12 \\
\mathrm{~mm}\end{array}$ \\
\hline $\begin{array}{l}\text { Material thermal } \\
\text { conductivity }\end{array}$ & $0.13 \mathrm{~W} \mathrm{~m}^{-1} \mathrm{~K}^{-1}$ & $0.20 \mathrm{~W} \mathrm{~m}^{-1} \mathrm{~K}^{-1}$ & $0.19 \mathrm{~W} \mathrm{~m}^{-1} \mathrm{~K}^{-1}$ \\
\hline $\begin{array}{l}\text { Number of } \\
\text { simultaneous } \\
\text { samples }\end{array}$ & 100 & & 9 \\
\hline $\begin{array}{l}\text { Reaction volume } \\
\text { per assay }\end{array}$ & $4 \mu \mathrm{L}$ & $25 \mu \mathrm{L}$ & $4 \mu \mathrm{L}$ \\
\hline $\begin{array}{l}\text { DNA amount per } \\
\text { assay }\end{array}$ & $2.56 \mathrm{ng}$ & $16 \mathrm{ng}$ & $2.56 \mathrm{ng}$ \\
\hline
\end{tabular}

\section{Analytical performance of the genotyping assay}

393 Having demonstrated that AS-RPA can be used for SNP genotyping in a single 394 format, the capabilities of a multiplex detection method were studied. Among 395 the techniques currently available (i.e. optical, electrochemical, etc.), AS-RPA 396 on PLA chips combined to a hybridization assay on PC chips was tested. This 397 detection approach showed excellent performance to simultaneously identify 398 several PCR products [10].

399 The AS-RPA products from the target genes related to the tobacco use disorder 400 were simultaneously determined. Selectivity was estimated from cross-reactivity 401 experiments by hybridizing products from single amplification assays on a chip 402 that contained probes for the five studied genes (four target genes and a control 403 gene) (Figure 3). Positive responses were obtained only for the specific probe, 404 and were negative for the remaining ones. Sensitivity was determined by 405 preparing heterozygous mixtures with increasing percentages of wild-type DNA 
<smiles>C1#C[Co]1</smiles> 
(a)

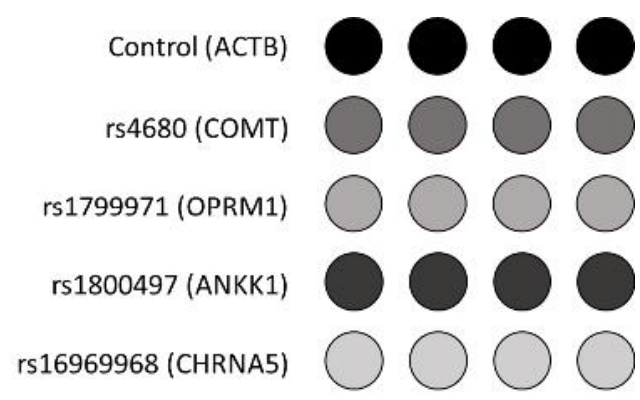

(b)

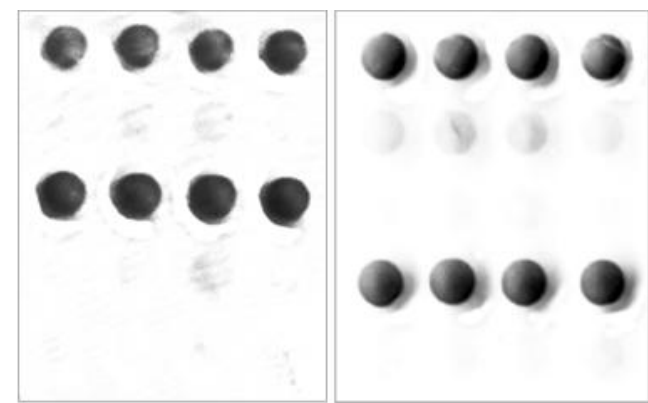

(c)

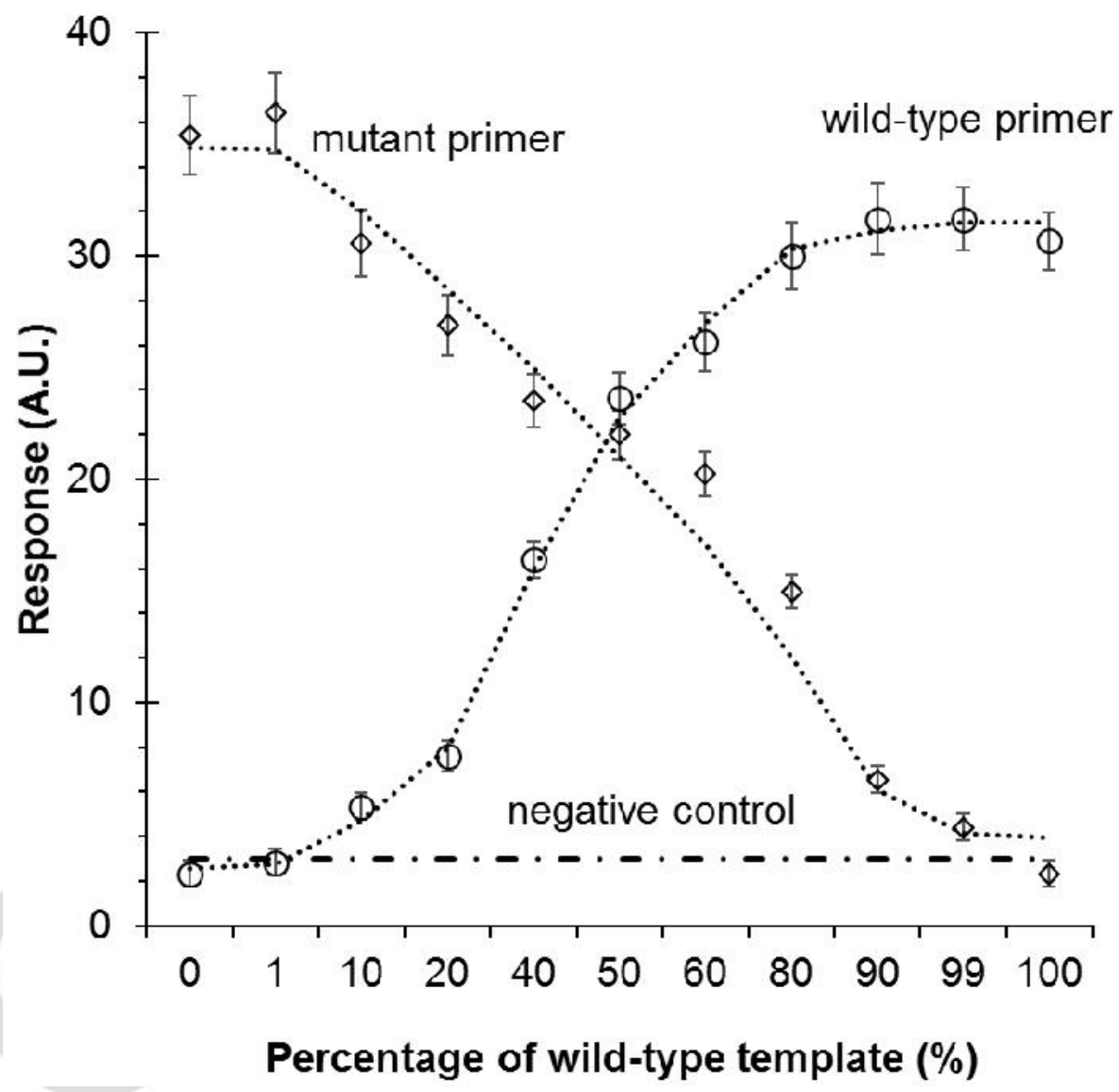

420 Figure 3. (a) Probe layout of a microarray chip (b) Microarray images obtained 421 for the amplification products: rs1799971 (left) and rs1800497 (right).(c) Assay 422 response depending on the percentage of wild-type template compared to the 423 total template for both RPA mixtures (wild-type FP and mutant FP). Logistic 424 regressions: $y=32 /(1+\exp (2.43-0.07 t), R=0.982$ for the wild-type and $y=$ $42537 /(1+\exp (-2.80+0.05 \mathrm{t}), \mathrm{R}=-0.935$ for the mutant. 


\section{Analysis of patient samples}

427 The applicability of chip-based AS-RPA for genotyping screening in tobacco 428 cessation treatment, or for drugs used in nicotine dependence, was 429 investigated. As a biological sample, buccal swab extracts were selected. In a 430 clinical scenario, the use of buccal swabs is noninvasive, less stressful and a 431 much easier technique to collect DNA samples. Moreover, sample storage does 432 not require refrigeration and DNA extraction is a much simpler process than 433 blood samples. As a detection strategy, a desktop scanner was used for chip 434 reading. Then the procedure was performed using low-resource laboratory 435 materials and equipment (i.e. a primary health center). The analysis time was $436210 \mathrm{~min}$ (DNA extraction: $50 \mathrm{~min}$, amplification: $60 \mathrm{~min}$, hybridization-detection: $437100 \mathrm{~min})$.

438 The absorption measurements indicated that a sufficient amount of high quality 439 amplifiable human DNA was isolated from all the tested samples. The reference 440 (ACTB gene) and targeted regions were amplified and submitted to on-chip 441 hybridization. Figure SI.3 illustrates some examples of the microarray images. 442 Presence of mutated variants in rs4680 and rs1799971 was detected. A 443 subsequent comparison of the acquired chip signals with patient stratification 444 based on the reference method clearly demonstrated perfect matching. The 445 clinical implications of the provided genotyping results are a review of drug 446 treatment, including anti-depressives or nicotine replacement products (e.g. 447 patch). Functional polymorphisms in dopamine pathways (rs4680) are 448 associated with the use of bupropion to mitigate lapsing to smoking following a 449 quit attempt [34]. Better prolonged abstinence rates have been reported after 450 the nicotine replacement therapy tailored to each smoker, and based on either 451 genotype in the opioid receptor (rs1799971) [26].

452 Presence of mutant alleles was detected for $70.6 \%$ (rs 4680 ), $52.9 \%$ 453 (rs1799971), $41.2 \%$ (rs1800497), and $41.2 \%$ (rs16969968) of the smoker 454 patients. A discrimination factor for genotype assignment was calculated from 455 the signal-to-noise ratios recorded in the microarray images (Figure 4). Each 456 call type within each target polymorphism statistically and significantly differed 457 from the others ( $p$-values <0.0001). Homozygous genotypes led to 
458 discrimination factors above 0.3 and under -0.3 for the wild-type and the 459 mutant, respectively. An intermediate discrimination factor (between -0.3 and $460+0.3)$ was calculated for each heterozygous genotype. The genotype 461 assignments, listed in Supplementary Material, agreed with those obtained by 462 the reference method (100\% coincidence).

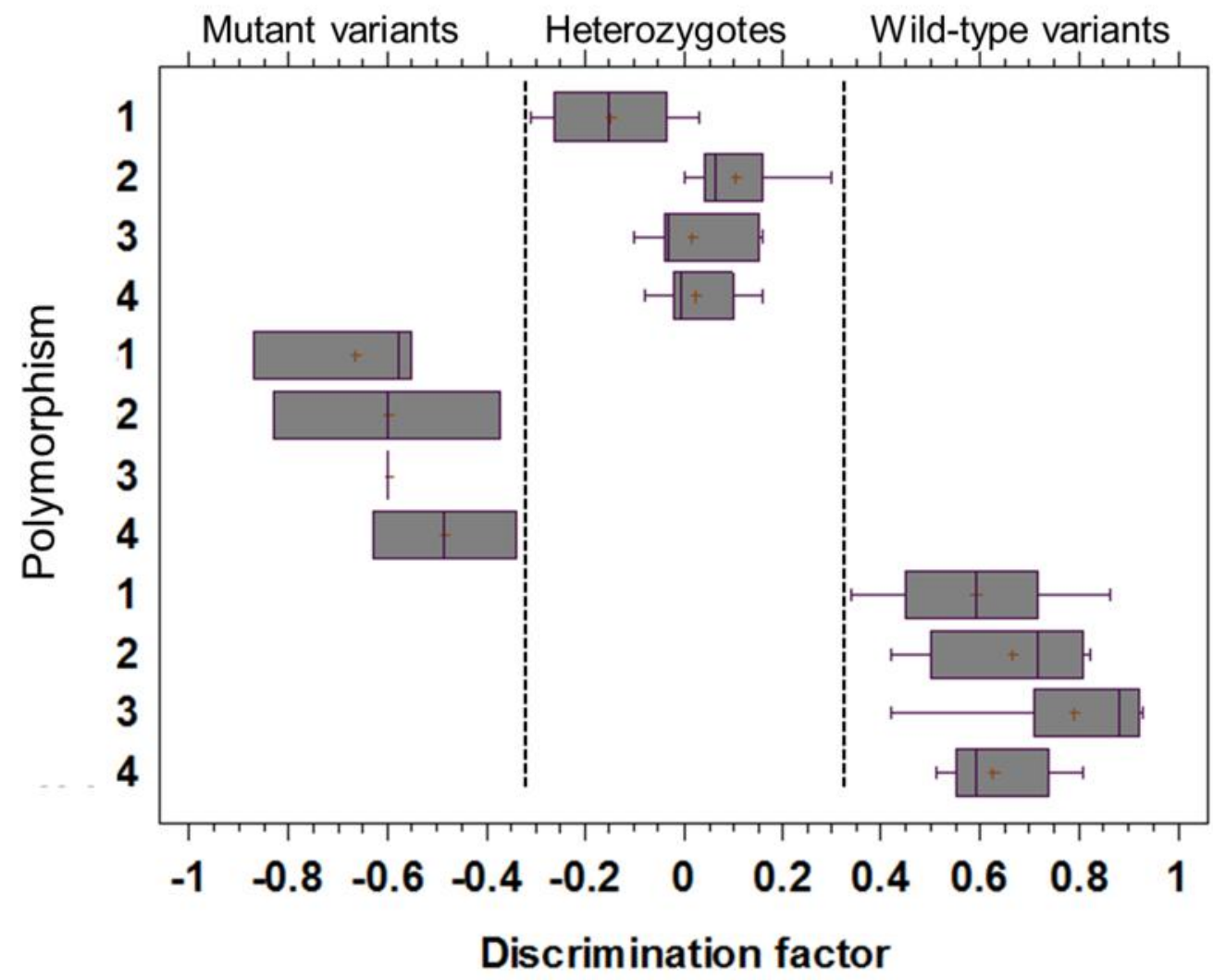

463

464 Figure 4. Boxplots of the discrimination factors classified according to the 465 polymorphism and population group. 1: rs4680, 2: rs1799971, 3: rs1800497, 466 and 4: rs 16969968 CONCLUSIONS

469 In the last few decades, patient genome information has been proposed to 470 select individual clinical care, particularly drug treatment decisions. However, 471 the impact of personalized medicine is low compared to the research advances 472 made. The results reported herein study demonstrate how pharmacogenomics 473 knowledge combined with emerging analytical methodologies can benefit 474 clinical practice more broadly. Although more in-depth research must be 
475 conducted, the combination of two innovative solutions was a success. Firstly, 476 the advantages of the isothermal amplification reaction were incorporated to 477 acquire the demanded copy number for sensitive SNP loci detection. The best 478 features were their fast-response (4-fold compared to the PCR), low 479 temperature $\left(37{ }^{\circ} \mathrm{C}\right)$ and few design restrictions. However, the reduced 480 multiplexing capabilities forced single parallelized reactions. Secondly, we 481 employed additive manufacturing based on using a 3D printer as the chosen 482 technology to create a customer-tailored platform for high-throughput analyses. 483 The 100-well PLA-chip design considerably reduced reagent consumption and 484 avoided expensive manufacturing processes or complex pumping systems associated with some DNA detection instruments. In fact the assay can be 486 performed with standard materials (i.e. pipettes, oven) found in clinical laboratories. The present work demonstrates that PLA is an adequate material for performing enzymatic reactions in a static format. The following step is to achieve better point-of-care performance and the next challenge is to develop microfluidic devices fabricated with this material that integrate all DNA assay steps, from extraction to detection. The advantages include the method's flexibility and accessibility compared to other micro-prototyping or microfabrication techniques.

494 Regarding the clinical impact, today pharmacogenomics is applied mainly to 495 certain treatments in psychiatry, oncology and cardiology. One main reason is 496 the cost-effectiveness of genotyping methods. In addition, only some diseases 497 apply to this approach because their treatment generally involves expensive 498 pharmaceutical products or drugs with highly probable adverse effects. With our 499 approach, personalized therapies based on incorporating genetics into patient 500 stratification can be offered, and even for relatively less-impact treatments. The 501 methodology's cost-effectiveness, flexibility and portability will support the well502 known genetic marker for predicting drug responses. In the particular case of 503 tobacco addiction, genotyping information will help predict the degree of 504 success in smoking cessation. 
507 The authors acknowledge the financial support received from the Generalitat

508 Valenciana (GVA-PROMETEOII/2014/040 Project and GRISOLIA/2014/024 $509 \mathrm{PhD}$ grant) and the Spanish Ministry of Economy and Competitiveness 510 (MINECO CTQ2013-45875-R project).

\section{REFERENCES}

512 (1) Manolio TA, Chisholm RL, Ozenberger B, Roden DM, Williams MS, 513 Wilson R, et al. (2013) Implementing genomic medicine in the clinic: the future 514 is here. Genet Med 15:258-267.

515 (2) Scott SA (2013) Clinical pharmacogenomics: Opportunities and 516 challenges at point-of-care. Clin Pharmacol Ther 93:33.

517 (3) Limaye N (2013) Pharmacogenomics, Theranostics and Personalized 518 Medicine-the complexities of clinical trials: challenges in the developing world. 519 Appl Transl Genomics 2:17-21.

520 (4) Abul-Husn NS, Owusu Obeng A, Sanderson SC, Gottesman O, Scott SA 521 (2014) Implementation and utilization of genetic testing in personalized 522 medicine. Pharmacogenomics Pers Med 7:227-40.

523 (5) Knez K, Spasic D, Janssen KP, Lammertyn J (2014) Emerging 524 technologies for hybridization based single nucleotide polymorphism detection. 525 Analyst 139:353-370.

526 (6) Shen W, Tian Y, Ran T, Gao Z (2015) Genotyping and quantification 527 techniques for single-nucleotide polymorphisms. TrAC Trends Anal Chem 69:152813.

529 (7) Milbury CA, Li J, Makrigiorgos GM (2009) PCR-based methods for the 530 enrichment of minority alleles and mutations. Clin Chem 55:632-640.

531 (8) Asari M, Watanabe S, Matsubara K, Shiono H, Shimizu K. (2009) Single 532 nucleotide polymorphism genotyping by mini-primer allele-specific amplification 533 with universal reporter primers for identification of degraded DNA. Anal 534 Biochem 386:85-90.

535 (9) Taira C, Matsuda K, Yamaguchi A, Sueki A, Koeda H, Takagi F, 536 Kobayashi Y, Sugano M, Honda T. (2013) Novel high-speed droplet-allele 537 specific-polymerase chain reaction: Application in the rapid genotyping of single 538 nucleotide polymorphisms. Clin Chim Acta, 424:39-46. 
539 (10) Tortajada-Genaro LA, Mena S, Niñoles R, Puigmule M, Viladevall L, 540 Maquieira A (2016)Genotyping of single nucleotide polymorphisms related to 541 attention-deficit hyperactivity disorder. Anal Bioanal Chem 408:2339-2345.

542 (11) Woolley CF, Hayes MA (2014) Emerging technologies for biomedical 543 analysis. Analyst139:2277-2288.

544 (12) Craw P, Balachandran W (2012) Isothermal nucleic acid amplification 545 technologies for point-of-care diagnostics: a critical review. Lab Chip, 12:24695462486.

547 (13) Zhang L, Zhang Y, Wang C, Feng Q, Fan F, Zhang G, Kang X, Qin X, 548 Sun J, Li Y, Jiang $X$ (2014) Integrated microcapillary for sample-to-answer 549 nucleic acid pretreatment, amplification, and detection. Anal Chem 86:1046155010466.

551 (14) Chen F, Zhao Y, Fan C, Zhao, Y (2015) Mismatch Extension of DNA 552 Polymerases and High-Accuracy Single Nucleotide Polymorphism Diagnostics 553 by Gold Nanoparticle-Improved Isothermal Amplification. Anal Chem 87:87185548723.

555 (15) Li J, Macdonald J (2015) Advances in isothermal amplification: novel 556 strategies inspired by biological processes. Biosens Bioelectron 64:196-211.

557 (16) Santiago-Felipe S, Tortajada-Genaro LA, Morais S, Puchades R, 558 Maquieira A (2014)One-pot isothermal DNA amplification-Hybridisation and 559 detection by a disc-based method. Sens Actuator B-Chem 204:273-281.

560 (17) Santiago-Felipe, S., Tortajada-Genaro, L. A., Puchades, R., Maquieira, 561 Á. (2016) Parallel solid-phase isothermal amplification and detection of multiple 562 DNA targets in microliter-sized wells of a digital versatile disc. Microchimica 563 Acta, 183:1195-1202.

564 (18) Tortajada-Genaro LA, Santiago-Felipe S, Amasia M, Russom A, 565 Maquieira A (2015) Isothermal solid-phase recombinase polymerase 566 amplification on microfluidic digital versatile discs (DVDs). RSCAdv 5:2998756729995.

568 (19) Li Z, Liu Y, Wei Q, Liu Y, Liu W, Zhang X, Yu Y (2016) Picoliter Well 569 Array Chip-Based Digital Recombinase Polymerase Amplification for Absolute 570 Quantification of Nucleic Acids. PloS one, 11:e0153359. 
571 (20) Daher RK, Stewart G, Boissinot M, Boudreau DK, Bergeron MG (2015) 572 Influence of sequence mismatches on the specificity of recombinase 573 polymerase amplification technology. Mol Cell Probes29:116-121.

574 (21) Shin Y, Perera AP, Kim KW, Park MK (2013) Real-time, label-free 575 isothermal solid-phase amplification/detection (ISAD) device for rapid detection 576 of genetic alteration in cancers. Lab Chip 13:2106-2114.

577 (22) NgePN, Rogers Cl, Woolley AT (2013) Advances in microfluidic 578 materials, functions, integration, and applications. Chem Rev 113:2550-2583.

579 (23) Bhattacharjee N, Urrios A, Kang S, Folch A (2016)The upcoming 3D580 printing revolution in microfluidics. Lab Chip 16:1720-1742.

581 (24) Waheed S, Cabot JM, Macdonald NP, Lewis T, Guijt RM, Paull B, 582 Breadmore MC (2016) 3D printed microfluidic devices: enablers and barriers. 583 Lab Chip 16:1993-2013.

584 (25) Bierut LJ, Madden PA, Breslau N, Johnson EO, Hatsukami D, Pomerleau 585 OF, Swan GE, Rutter J, Bertelsen S, Fox L, Fugman D, Goate AM, Hinrichs AL, 586 Konvicka K, Martin NG, Montgomery GW, Saccone NL, Saccone SF, Wang JC, 587 Chase GA, Rice JP Ballinger DG (2007) Novel genes identified in a high-density 588 genome wide association study for nicotine dependence. Hum MolGen 16:2458935.

590 (26) Carpenter MJ, Jardin BF, Burris JL, Mathew AR, Schnoll RA, Rigotti NA, 591 Cummings KM (2013) Clinical strategies to enhance the efficacy of nicotine 592 replacement therapy for smoking cessation: a review of the literature. Drugs, 593 73:407-426.

594 (27) Moody C, Newell H, Viljoen H (2016) FA mathematical model of 595 recombinase polymerase amplification under continuously stirred conditions. 596 Biochem Eng J 112:193-201.

597 (28) Dimitrov RA, Zuker M (2004) Prediction of hybridization and melting for 598 double-stranded nucleic acids. Biophys J 87:215-226.

599 (29) Zhang C, Xing D (2007) Miniaturized PCR chips for nucleic acid 600 amplification and analysis: latest advances and future trends. Nucleic Acids Res $601 \quad 35: 4223-4237$. 
602 (30) Liu B, Huang PJJ, Zhang X, Wang F, Pautler R, IpACF, Liu J (2013) 603 Parts-per-million of polyethylene glycol as a non-interfering blocking agent for 604 homogeneous biosensor development. Anal Chem 85:10045-10050.

605 (31) Wu J, Kodzius R, Cao W, Wen W (2014) Extraction, amplification and 606 detection of DNA in microfluidic chip-based assays. Microchim Acta 181:16116071631.

608 (32) Li J, Wang L, Mamon H, Kulke MH, Berbeco R, Makrigiorgos GM (2008) 609 Replacing PCR with COLD-PCR enriches variant DNA sequences and 610 redefines the sensitivity of genetic testing. Nat Med 14:579-84.

611 (33) Shen R, Fan JB, Campbell D, Chang W, Chen, J., Doucet, D., Yeakley J, 612 Bibikova M, Garcia EW, McBride C, Steemers F, Garcia F, Kermani BG, 613 Gunderson K, Oliphant A (2005) High-throughput SNP genotyping on universal 614 bead arrays. Mut Res Fund Mol M 573:70-82.

615 (34) David SP, Strong DR, Leventhal AM, Lancaster MA, McGeary JE, 616 Munafò MR, Bergen AW, Swan GE, Benowitz NL, Tyndale RF, Conti DV, 617 Brown RA, Lerman C, Niaura R (2013) Influence of a dopamine pathway 618 additive genetic efficacy score on smoking cessation: results from two 619 randomized clinical trials of bupropion. Addiction 108:2202-2211. 